\title{
HOMBRES (1950) DE FRED ZINNEMANN: PARAPLEJÍA Y PRINCIPALES PROTAGONISTAS EN LA REHABILITACIÓN DE UNA DISCAPACIDAD
}

\section{The Men (1950) by Fred Zinnemann: Paraplegia and main protagonists in the rehabilitation of a disability}

\author{
Palmer José HERNÁNDEZ-YÉPEZ \\ Facultad de Medicina Humana. Universidad Privada Norbert Wiener (Perú). \\ e-mail: palmerxvii@gmail.com
}

Fecha de recepción: 2 de enero de 2020

Fecha de aceptación: 16 de enero de 2020

Fecha de publicación: 15 de septiembre de 2020

\begin{abstract}
Resumen
Hombres (1950) de Fred Zinnemann y protagonizada por Marlon Brando, es una película que cuenta la historia del teniente Ken Wilcott, quien sufre de paraplejía producto de una herida de bala en la espalda cuando se encontraba de servicio en la Segunda Guerra Mundial. Con la ayuda del Dr. Brock y su prometida Ellen logra sobreponerse a su discapacidad e iniciar su proceso de aceptación y rehabilitación. A través del filme se puede apreciar el proceso que padecen los pacientes con alguna discapacidad, desde la negación y depresión hasta finalmente aceptar su discapacidad y proponerse a establecer un buen futuro. Asimismo, en el presente trabajo se discute la importancia de los principales personajes y sus funciones en torno a una discapacidad: el paciente, el médico, la familia y la sociedad.
\end{abstract}

Palabras clave: discapacidad; paraplejía; rehabilitación; discriminación.

\section{Abstract}

The Men (1950) by Fred Zinnemann and starring Marlon Brando, is a movie that shows the story of Lieutenant Ken Wilcott, who suffers from paraplegia due to a gunshot wound in the back when he was on duty in World War II. With the help of Dr. Brock and his fiancée Ellen he manages to overcome his disability and begin his process of acceptance and rehabilitation. Through the film we can see the process suffered by patients with disabilities, from denial and depression to 


\section{HOMBRES (1950) DE FRED ZINNEMANN: PARAPLEJÍA Y PRINCIPALES PROTAGONISTAS EN LA REHABILITACIÓN DE UNA DISCAPACIDAD \\ PALMER JOSÉ HERNÁNDEZ-YÉPEZ}

finally accept their disability and set out to establish a good future. In the same way, we discuss the importance of the main characters and their functions around a disability: the patient, the doctor, the family and society.

Key words: disability; paraplegia; rehabilitation; discrimination.

\section{Ficha técnica}

Título original: The Men.

Otros títulos: Hombres (España).

País: Estados Unidos.

Año: 1950.

Director: Fred Zinnemann.

Música: Dimitri Tiomkin.

Fotografía: Robert De Grasse.

Montaje: Harry Gerstad.

Guion: Carl Foreman.

Intérpretes: Marlon Brando, Teresa Wright, Everett Sloane, Jack Webb, Richard Erdman, Arthur Jurado, Virginia Farmer, Dorothy Tree, Howard St. John, Nita Hunter, Patricia Joiner, ...

Color: blanco y negro.

Duración: 85 minutos.

Género: drama, discapacidad.

Idioma original: inglés.

Productoras: Stanley Kramer Productions.

Sinopsis: "Kenneth Wilcott (Marlon Brando), es otro oficial del ejército estadounidense que ha entrado a engrosar las filas de discapacitados que ha dejado la Segunda Guerra Mundial. Tras el fin de la guerra, Ken y otros veteranos, seguirán batallando para superar su drama de la mejor manera» (FilmAffinity).

\section{Enlaces:}

https://www.imdb.com/title/tt0042727

https://www.filmaffinity.com/es/film944431. html

\section{Tráiler en inglés}

\section{Introducción}

La paraplejía es la pérdida severa o completa de la función motora en las extremidades inferiores debido a una lesión medular producto principalmente de un traumatismo. La etiología es encabezada por accidentes automovilísticos, caídas, deportes de contacto y por heridas de arma de fuego. Este último factor está en constante aumento, anteriormente por los conflictos bélicos existentes y hoy en día por el aumento de la violencia e inseguridad en las ciudades.

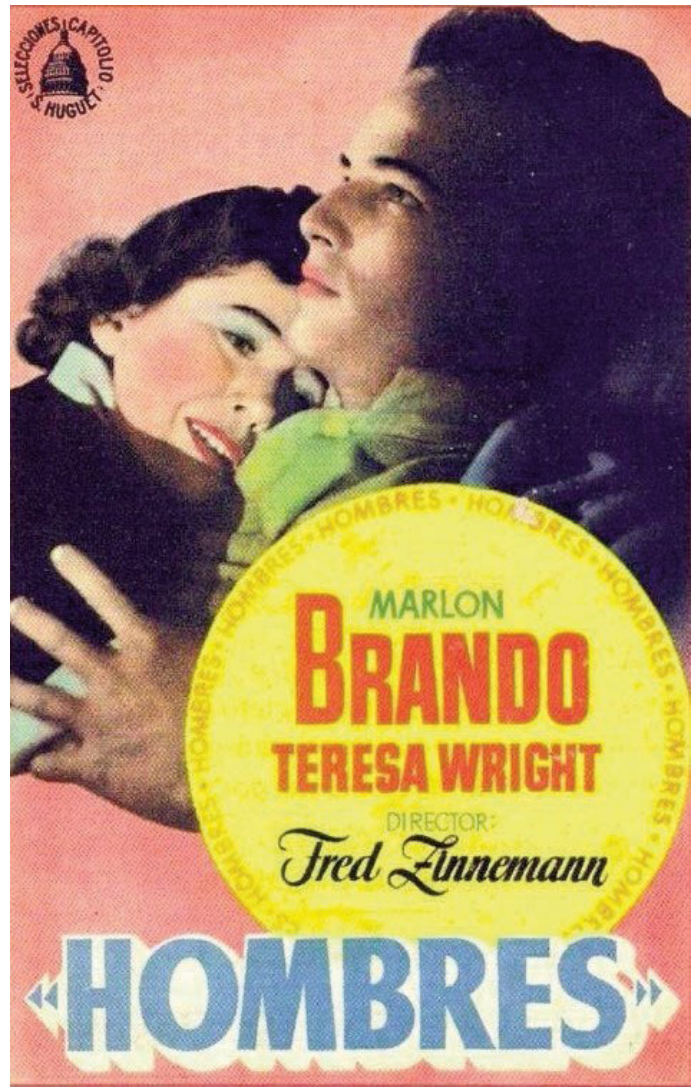

Cartel español.

Rev. Med. Cine. 2020; 16(3), 185-191 Ediciones Universidad de Salamanca / 요

J. Med. Mov., 2020; 16 (3), 185-191 


\section{HOMBRES (1950) DE FRED ZINNEMANN: PARAPLEJÍA Y PRINCIPALES PROTAGONISTAS EN LA REHABILITACIÓN DE UNA DISCAPACIDAD PALMER JOSÉ HERNÁNDEZ-YÉPEZ}

El filme Hombres (1950) de Fred Zinnemann gira en torno a la vida del teniente Ken Wilcott en su regreso a su país después de quedar paralítico por un disparo de bala en la Segunda Guerra Mundial (Foto 1). El teniente Wilcott, interpretado por Marlon Brando, sufre todos los sucesos que desencadena un trauma de tal magnitud, desde la negación, ira, depresión y posterior aceptación. El personaje tiene la intriga de no poder realizar una vida "normal», y por lo tanto, al inicio, no desea realizar ningún tipo de rehabilitación física rindiéndose a la postración continua acompañada de ira y desilusión por la vida.

Posteriormente con la ayuda del doctor Brock y de su prometida Ellen, logra aceptar su discapacidad y sobreponerse a ello, cumpliendo con una estricta rehabilitación física, fortaleciendo su condición psicológica y sonriéndole a la vida. Como resultado, logra el alta médica, se casa con Ellen y construyen su propio hogar.

En este artículo, se propone reflexionar sobre los temas concernientes a la paraplejía, desde el aspecto médico, abordando su etiología, clínica y principales complicaciones; el aspecto psicológico, pues es un trauma para el paciente entender y aceptar que no volverá a usar sus piernas; por último sobre los principales personajes que intervienen, el paciente, el médico, la familia y la sociedad desde el punto de vista propuesto en la película.

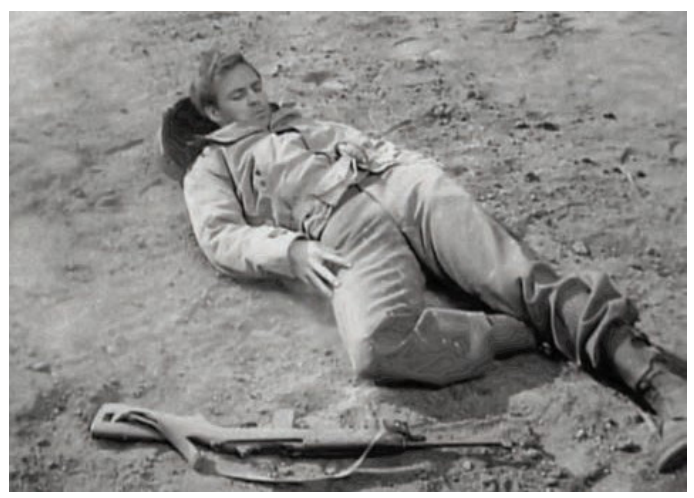

Foto 1. El teniente Ken Wilcott recibe un disparo en la espalda quedando tendido en el suelo.

\section{La paraplejía}

La paraplejía es la pérdida severa o completa de la función motora en las extremidades inferiores y en las porciones inferiores del tronco como resultado de una lesión medular o por una enfermedad congénita ${ }^{1}$. El trauma penetrante representa del $13 \%$ al $17 \%$ de todas las lesiones de la columna ${ }^{2}$. En Estados Unidos, la causa más común de lesión de la médula espinal son los accidentes de tránsito seguido de una caída desde una altura. En tercer lugar, se encuentran las lesiones por armas de fuego. Se debe resaltar que las lesiones de médula espinal debido a heridas de bala ocurren como resultado de efectos traumáticos directos ${ }^{3}$.

El dolor es probablemente la complicación más común a largo plazo y es bastante frecuente en los casos de afectación de la cola de caballo y el cono medular. La eliminación de la bala no está asociada con la resolución del dolor. Generalmente se maneja con medicamentos, como los antidepresivos tricíclicos y los anticonvulsivantes ${ }^{4}$. Otro problema relacionado con la bala alojada es la toxicidad por metal, particularmente del plomo. La intoxicación sistémica por plomo, también llamado, saturnismo, es una complicación rara, pero puede suceder en casos que la bala permanece alojada en la columna vertebral ${ }^{5}$. La meningitis es una complicación inherente al trauma y el tratamiento quirúrgico se considera un factor de riesgo. La vejiga neurogénica e infección del tracto urinario son otras complicaciones comunes. Otra posible complicación es la migración del proyectil, que puede o no causar cambios en la función neurológica ${ }^{6}$.

\section{Sobre el paciente}

La paraplejía supone una discapacidad física grave la cual afectará la calidad de vida de los pacientes. Esta condición se asocia con niveles anormales de morbilidad psicológica, abuso de sustancias y riesgo de suicidio ${ }^{7}$. No es de extrañar

Rev. Med. Cine. 2020; 16(3), 185-191 Ediciones Universidad de Salamanca / @®@ J. Med. Mov., 2020; 16 (3), $185-191$ 
tal comportamiento debido a la ansiedad que considera no volver a poder emplear las piernas. En el filme, el teniente Wilcott medita para sí mismo: "Tenía miedo de morir y ahora miedo de vivir».

Según el modelo Kübler-Ross ${ }^{8}$, existen cinco fases del duelo: negación, ira, negociación, depresión y aceptación; y no necesariamente se cumplen todas las fases y el orden propuesto. Aunque este modelo es más característico en pacientes terminales también puede ajustarse para pacientes que hayan sufrido una pérdida considerable (en este caso el caminar).

Se observa una evolución en el pensamiento del teniente Wilcott: "La guerra ha acabado, y me alegro de estar un medio vivo. Soy un tipo con suerte». Esto traduce la etapa de negociación, en la que se acepta parcialmente la situación con condiciones y se tiene una esperanza en aplazar la muerte. En cuanto a la etapa de aceptación, no necesariamente se consolida con palabras, sino con acciones. Se puede observar que el teniente acepta su discapacidad, pero a la vez entiende que no necesariamente tiene por qué ser una incapacidad, por lo que inicia con sus ejercicios físicos como parte del esquema de rehabilitación (Foto 2), empieza a ser más sociable con los compañeros de la sala de hospitalización, acepta el hecho que deberá usar una silla de ruedas por el resto de su vida, retoma el contacto con su prometida (a quien él había apartado por vergüenza de no poder caminar) y finalmente se casan.

Es importante tener en cuenta, que la etapa de aceptación no llegará rápido y tampoco significa que se generará de forma espontánea. Es la combinación de varios factores psicológicos que afianzan el apoyo en el paciente, en el cual la familia, el equipo médico y los amigos juegan un rol importante.

\section{Sobre la figura del médico}

La paraplejía abarca un dolor físico continuo y también un constante estrés emocional con cambios en el plano afectivo, cognitivo y en el comportamiento. Si bien el dolor físico (y las complicaciones de la paraplejía) pueden manejarse con cirugía o farmacoterapia, el factor emocional sigue siendo clave en la recuperación completa y global del paciente.

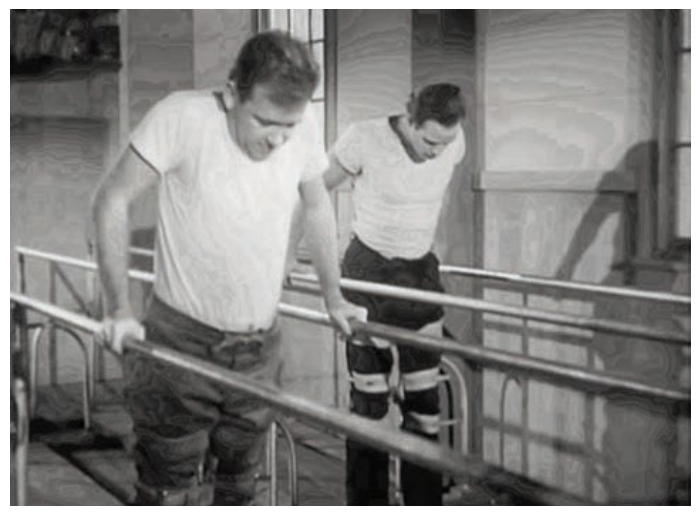

Foto 2. El teniente Wilcott inicia sus ejercicios del programa de rehabilitación física.

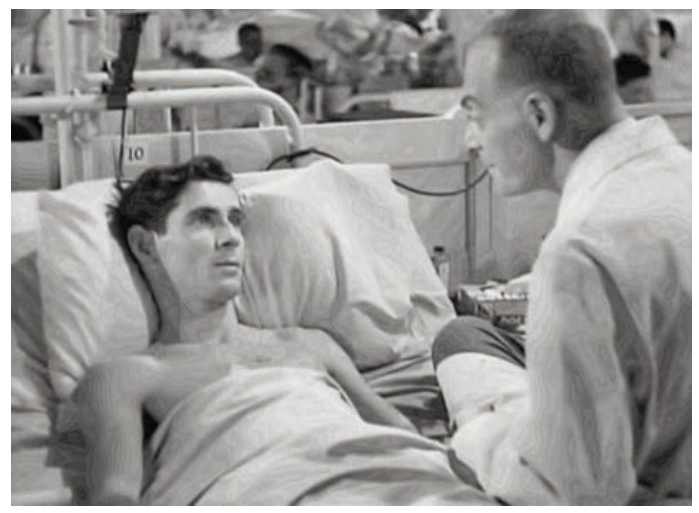

Foto 3. El Dr. Brock brinda tranquilidad y confianza en los pacientes con paraplejía.

Un rol importante en esa recuperación es la confianza que se puede forjar entre el paciente y el médico. Y es que la relación médico-paciente cuando es correcta se considera un tipo de amistad, la amistad médica, en donde el médico aporta su saber e intención de ayuda y el paciente aporta su confianza y esperanza en el médico que lo atiende ${ }^{9}$. Una relación médico-paciente 


\section{HOMBRES (1950) DE FRED ZINNEMANN: PARAPLEJÍA Y PRINCIPALES PROTAGONISTAS \\ EN LA REHABILITACIÓN DE UNA DISCAPACIDAD \\ PALMER JOSÉ HERNÁNDEZ-YÉPEZ}

siguiendo los preceptos de la atención centrada en el paciente es un recurso importante que puede conducir a una mayor capacidad de recuperación y resiliencia del paciente ${ }^{10}$.

No obstante, es menester resaltar que el manejo de un paciente con lesión medular y paraplejía es multidisciplinario, en donde diferentes doctores especialistas (neurólogo, neurocirujano, fisiatra, psiquiatra), enfermeras, rehabilitadores, etc. todos tienen un papel importante en la evolución del paciente. No sería de extrañar que en algún momento se establezca un tipo de relación interpersonal, ya no la relación dualista médicopaciente, sino una relación del equipo médico y el paciente.

En el filme, el doctor encargado del proceso de rehabilitación de los pacientes con herida de bala y posterior afectación de la médula espinal es el Dr. Brock, quien en las visitas médicas no solo resalta los asuntos estrictamente médicos, sino que abarca temas personales con los pacientes como sus proyectos de vida después de la guerra, el futuro, sus estudios, sus familias, etc. (Foto 3 ). Se forja así una relación sólida con los pacientes ganándose su carisma y confianza. «Estamos orgullosos de usted», "¿Cómo va la familia?», «¿Ha decidido que es lo que va a hacer cuando se vaya?» o "Con paciencia todo llega en esta vida» son algunas de las frases que se le escucha al Dr. Brock a lo largo de la visita médica motivando a sus pacientes a adherirse y colaborar con el tratamiento de rehabilitación y tener un propósito que los motive a seguir adelante.

\section{Sobre la familia}

Un rol igual de importante cumple la familia, pues es la entidad más cercana por naturaleza al paciente. En un estudio se demostró la importancia del compromiso por parte de los familiares en la recuperación de los pacientes con dolor crónico ${ }^{11}$. Los familiares tienen que estar siempre atentos a las necesidades del paciente, por ejemplo, en sus actividades de la vida diaria, las cuales algunas de ellas requerirán apoyo. Asimismo, deben tener atención sobre los signos de alarma de posibles complicaciones e instruirse sobre la condición que tiene el paciente. La familia constituye el soporte emocional en la recuperación del paciente, por lo tanto, se debe fortalecer la comunicación entre los miembros.

En el filme se aprecia como los familiares de los pacientes, especialmente las esposas, se comprometen en la discapacidad que poseen los pacientes parapléjicos, acudiendo a las charlas informativas por parte del Dr. Brock (Foto 4). Las preguntas formuladas por los familiares se centran en una posible cura, edad, los hijos, el comportamiento del paciente. La edad es una interrogante frecuente en la paraplejía puesto que en la película (y en la vida real) la mayoría de los pacientes son jóvenes adultos. "Mi hijo tiene 19 años». le dice una madre preocupada al Dr. Brock y él responde: «Lo sé, pero si se cuida puede llegar a los 90». Otra pregunta formulada por una esposa es: «¿Es posible tener otro hijo?» respondiendo el doctor: "Es difícil de decir, en muchos casos es posible». Otra interrogante es: "Quiero seguir con mi marido, pero ha cambiado tanto, es otro». El Dr. Brock responde: «No, no es otro. Es el mismo hombre, ahora con una lesión en la espina, solo que está deprimido (...) Todo es cuestión de tiempo y de paciencia».

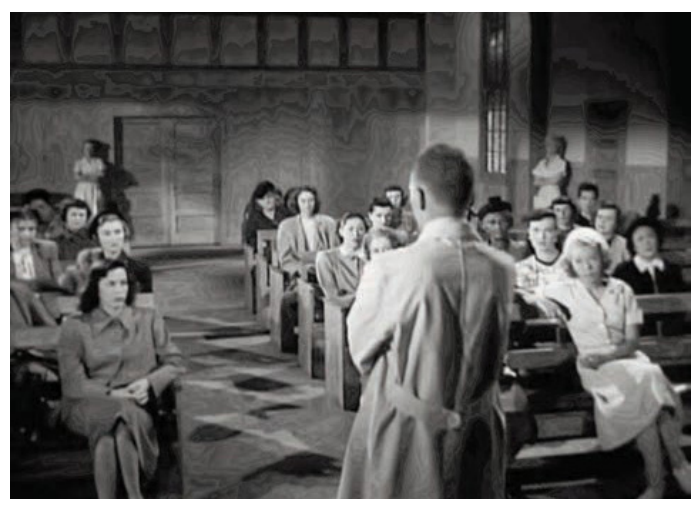

Foto 4. Familiares de los pacientes recibiendo recomendaciones por parte del Dr. Brock.

Rev. Med. Cine. 2020; 16(3), 185-191 Ediciones Universidad de Salamanca / @®@ J. Med. Mov., 2020; 16 (3), $185-191$ 


\section{HOMBRES (1950) DE FRED ZINNEMANN: PARAPLEJÍA Y PRINCIPALES PROTAGONISTAS EN LA REHABILITACIÓN DE UNA DISCAPACIDAD \\ PALMER JOSÉ HERNÁNDEZ-YÉPEZ}

\section{Sobre la sociedad}

Es un hecho que la persona con discapacidad, al igual que cualquier otra persona, es un producto de su interacción social con el entorno. Es decir, la sociedad tiene un papel fundamental en la recuperación y reintegración del paciente ${ }^{12}$. En el caso particular de las personas con lesión medular completa, al no poder utilizar sus piernas, se verán en la obligación de emplear una silla de ruedas para su desplazamiento. ¿Están la mayoría de las ciudades acondicionadas para el adecuado traslado de las personas en sillas de ruedas? Es un obstáculo el uso de medios de transportes como el bus, tranvía o metro, que, en la mayoría de los casos (al menos en los países en vías de desarroIlo), no tienen una rampa que permita el acceso de estas personas. A esto se suma la indiferencia de los conductores de estos servicios que instan a la persona a tomar un taxi porque les resultaría más fácil, generando mayor gasto económico en el paciente, que aparte de su tratamiento médico, también tiene que velar por su desplazamiento. ¿Las aceras tienen rampa de acceso para silla de ruedas? De igual forma, que el sistema de transporte, las principales vías de desplazamiento peatonal deben cumplir con este requisito y a la vez estar libres de cualquier obstáculo que dificulte el correcto movimiento de las personas con discapacidad, por ejemplo, postes de alumbrado público en mitad de la acera.

No menos importante es la sensibilidad de las personas. Es necesario hacer un análisis del comportamiento colectivo frente a las personas con algún tipo de discapacidad ya sea física, sensorial o intelectual. La justicia es lograda al momento que todos son reconocidos como iguales en la dignidad. Sin embargo, la justicia también es equidad. No se debe tratar a todos por igual cuando de por sí ya somos diferentes. Eso significaría una enorme injusticia. La persona con discapacidad, los enfermos, incluso los ancianos exigen un trato diferenciado por la situación de necesidad que poseen ${ }^{13}$. En últimas décadas se ha contemplado el surgimiento de organizaciones y grupos de apoyo que impulsan la integración de personas con alguna discapacidad. Adicionalmente, se han creado y fortalecido leyes que promueven y protegen las condiciones de igualdad en derechos de las personas con discapacidad consolidando así su inclusión efectiva en la sociedad. El concepto de integración es plasmado en el filme a través de la ayuda que se brindan los pacientes miembros de la Asociación de Veteranos Paralizados (AVP) y también cuando el teniente Wilcott se casa con su prometida Ellen y juntos construyen su casa, sentando las bases para un nuevo futuro (Fotos 5 y 6 ).

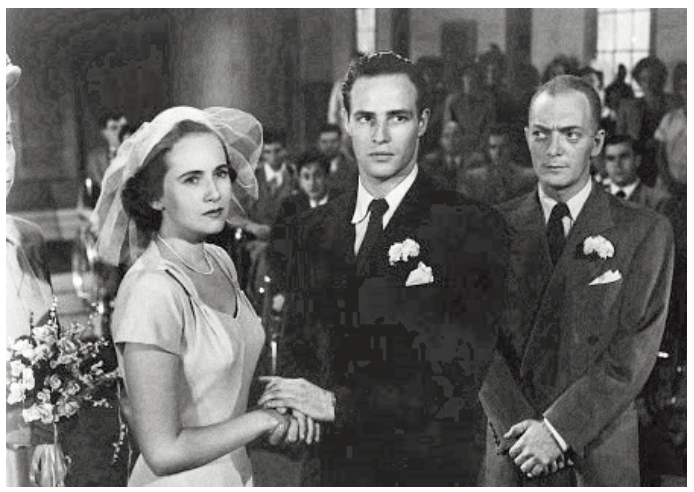

Foto 5. El teniente Wilcott se casa con su prometida Ellen. El Dr. Brock es el padrino.

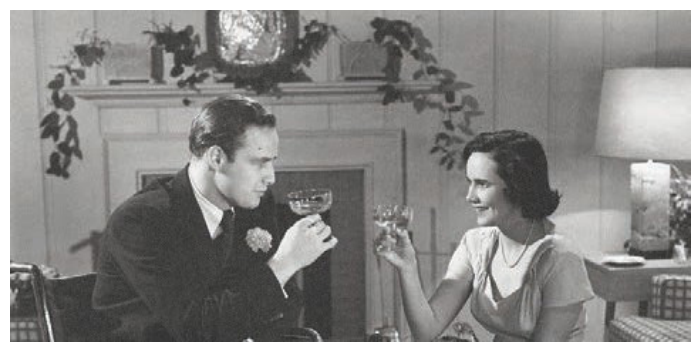

Foto 6. Ambos protagonistas inician su vida de casados brindando con esperanza por un gran futuro.

\section{Conclusión}

El ser humano es fascinante al poder hospedar contrastes de luces y sombras. ¿Persiste todavía

Rev. Med. Cine. 2020; 16(3), 185-191 Ediciones Universidad de Salamanca / @@ J. Med. Mov., 2020; 16 (3), $185-191$ 


\section{HOMBRES (1950) DE FRED ZINNEMANN: PARAPLEJÍA Y PRINCIPALES PROTAGONISTAS EN LA REHABILITACIÓN DE UNA DISCAPACIDAD \\ PALMER JOSÉ HERNÁNDEZ-YÉPEZ}

el estigma de discriminación frente a las personas con discapacidad? ¿Se sigue asociando el concepto que discapacidad es igual que incapacidad? La única forma de cambiar este pensamiento es a través de la educación, la cual debe ser integral, tolerante y que promueva la paz. El filme Hombres (1950) logra transmitir ese camino que pasan las personas que sufren alguna discapacidad, en este caso, la paraplejía. Debemos reflexionar sobre la indiferencia que persiste frente a las personas con discapacidad, lograr mantener y fortalecer nuestra humanidad, aquella capacidad que nos permite sentir afecto, comprensión y solidaridad hacia las demás personas. Y es en estos tiempos, gobernado por las redes sociales e imperado por la superficialidad y apariencia la cual vuelve a la sociedad más frívola, en donde los valores se necesitan más que nunca, dejando de ser un concepto ideal para que se conviertan en una realidad.

\section{Referencias}

1. Hidalgo-Martinez A. La rehabilitación terapéutica a pacientes parapléjicos: impacto desde las tecnologías. PODIUM-Revista de Ciencia y Tecnología en la Cultura Física. 2017; 12(1): 21-30.

2. de Barros Filho TE, Cristante AF, Marcon RM, Ono A, Bilhar R. Gunshot injuries in the spine. Spinal Cord. 2014; 52(7): 504-10.

3. Devivo MJ. Epidemiology of traumatic spinal cord injury: trends and future implications. Spinal Cord. 2012; 50(5): 365-72.

4. Spaić M, Petković S, Tadić R, Minić L. DREZ surgery on conus medullaris (after failed implantation of vascular omental graft) for treating chronic pain due to spine (gunshot) injuries. Acta Neurochir (Wien). 1999; 141(12): 1309-12.

5. Cristante AF, de Souza FI, de Barros Filho TE, Oliveira RP, Marcon RM. Lead poi- soning by intradiscal firearm bullet: a case report. Spine (Phila Pa 1976). 2010; 35(4): E140-3.

6. Aarabi B, Alibaii E, Taghipur M, Kamgarpur A. Comparative study of functional recovery for surgically explored and conservatively managed spinal cord missile injuries. Neurosurgery. 1996; 39(6): 1133-40.

7. Post, MWM, van Leeuwen, CMC. Psychosocial issues in spinal cord injury: a review. Spinal Cord. 2012; 50(5): 382-89.

8. Miaja-Avila M, Moral de la Rubia J. El significado psicológico de las cinco fases del duelo propuestas por Kübler-Ross mediante las redes semánticas naturales. Psicooncología. 2013; 10 (1): 109-30.

9. Mendoza A. La relación médico paciente: consideraciones bioéticas. Rev Peru Ginecol Obstet. 2017; 63(4): 555-64.

10. Náfrádi L, Kostova Z, Nakamoto K, Schulz PJ. The doctor-patient relationship and patient resilience in chronic pain: A qualitative approach to patients' perspectives. Chronic Illn. 2018; 14(4): 256-70.

11. Mawdsley L, Twiddy $H$, Longworth $M$. Involvement of Family and Friends in Pain Management Interventions. Journal of Observational Pain Medicine. 2015; 1(5): 12-21.

12. Kurawa SS. The impact of disability on self and society: an agenda for research on rehabilitation of disabled in Nigeria. Procedia Social and Behavioral Sciences. 2010; 5: 1804-10.

13. Ortega-Ruiz P. La construcción de la paz. Revista Virtual Redipe. 2016; 5(13): 17-35.

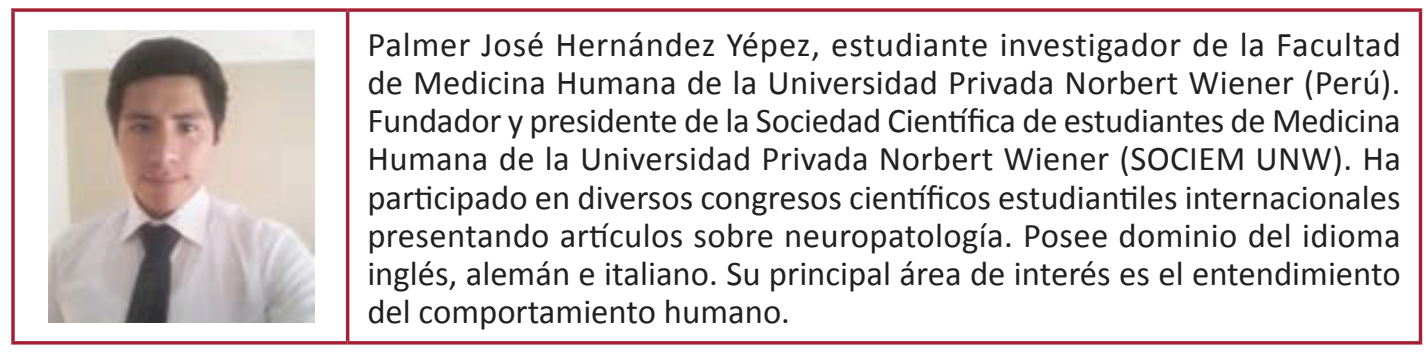

Rev. Med. Cine. 2020; 16(3), 185-191 Ediciones Universidad de Salamanca / @®@@

J. Med. Mov., 2020; 16 (3), 185-191 